\title{
The Hippo effector TAZ promotes cancer stemness by transcriptional activation of SOX2 in head neck squamous cell carcinoma
}

\author{
Jin $\mathrm{Li}^{1}$, Zhongwu $\mathrm{Li}^{1}$, Yaping $\mathrm{Wu}^{1}$, Yanling Wang ${ }^{1}$, Dongmiao Wang ${ }^{2}$, Wei Zhang ${ }^{3}$, Hua Yuan², Jinhai Ye ${ }^{2}$, \\ Xiaomeng Song ${ }^{2}$, Jianrong Yang ${ }^{2}$, Hongbing Jiang ${ }^{2}$ and Jie Cheng $\mathbb{1}^{1,2}$
}

\begin{abstract}
The Hippo-TAZ signaling has emerged as a fundamental regulator underlying cancer stem cells (CSCs) stemness which intricately associates with local recurrence and metastatic spreading in head neck squamous cell carcinoma (HNSCC). However, the precise downstream targets of TAZ responsible for HNSCC CSCS maintenance remain largely underexplored. Here, we identified Sex determining region Y box 2 (SOX2) as a putative downstream target of TAZ to promote CSCs maintenance and tumorigenicity in HNSCC. Both TAZ and SOX2 were significantly enriched in CSCs subpopulation $\left(\mathrm{CD}_{4} 4^{+} \mathrm{CD} 133^{+}\right)$isolated from Cal27 and Fadu cells via fluorescence-activated cell sorting. TAZ knockdown significantly reduced expression of SOX2 at both mRNA and protein levels, whereas its ectopic overexpression markedly increased its abundance in HNSCC cells. Moreover, reintroduction of ectopic SOX2 abolished, at least in part, the reduced tumorsphere formation and tumorigenicity in vivo induced by TAZ knockdown. Mechanistically, transcriptional complex formed by TAZ and TEAD4 was recruited to two binding sites in SOX2 promoter, which in turn facilitated transcription of SOX2 in HNSCC cells. In addition, the abundance of TAZ and SOX2 was positively correlated in HNSCC clinical samples, and both upregulations of TAZ and SOX2 associated with the worst survival. Taken together, our data reveal a previously unknown mechanistic linkage between TAZ and SOX2 and identify SOX2 as a direct downstream target of TAZ in modulating CSCS self-renewal and maintenance in HNSCC. These findings suggest that targeting TAZ-SOX2 axis might be a promising therapeutic strategy for HNSCC.
\end{abstract}

\section{Introduction}

Head and neck cancer comprises a heterogeneous group of malignancies that arise in the mucosal surfaces of the upper aerodigestive tract including oral and nasal cavity, pharynx and larynx as well as paranasal sinus ${ }^{1}$. The overwhelming majority of these tumors are squamous cell

Correspondence: Jie Cheng (jiecheng_njmu@163.com)

${ }^{1}$ Jiangsu Key Laboratory of Oral Disease, Nanjing Medical University, Nanjing 210029, PR China

${ }^{2}$ Department of Oral and Maxillofacial Surgery, Affiliated Stomatological Hospital, Nanjing Medical University, Nanjing 210029, PR China

Full list of author information is available at the end of the article.

These authors contributed equally: Jin Li, Zhongwu Li

Edited by B. Zhivotovsky carcinoma (SCC) and account for approximately $3.8 \%$ of all cancer cases and $3.6 \%$ of cancer-related deaths worldwide $^{2}$. Several etiological factors like tobacco smoking, alcohol consumption, chewing of betel quid and human papillomavirus (HPV) infection have been identified for HNSCC. Current clinical management of HNSCC including ablative surgery, radiotherapy and chemotherapy has yielded remarkable progress in the past decades. However, the long-term survival rate remains dismal and only $40-50 \%$ of patients survive for more than 5 years since initial diagnosis ${ }^{3}$. Molecular targeted therapies to treat advanced, recurrent or metastatic HNSCC are lacking or with limited success in selected patients ${ }^{4}$.

\section{(c) The Author(s) 2019}

(c) (i) Open Access This article is licensed under a Creative Commons Attribution 4.0 International License, which permits use, sharing, adaptation, distribution and reproduction in any medium or format, as long as you give appropriate credit to the original author(s) and the source, provide a link to the Creative Commons license, and indicate if changes were made. The images or other third party material in this article are included in the article's Creative Commons license, unless indicated otherwise in a credit line to the material. If material is not included in the article's Creative Commons license and your intended use is not permitted by statutory regulation or exceeds the permitted use, you will need to obtain permission directly from the copyright holder. To view a copy of this license, visit http://creativecommons.org/licenses/by/4.0/. 
These challenges largely hinge on our incomplete understanding about molecular tumorigenesis of HNSCC as well as its genetic and biological heterogeneities. Thus, in-depth investigations of molecular pathways underlying HNSCC pathogenesis will lead to novel effective therapeutics and optimal treatment guiding, ultimately improving patients survival and quality of life ${ }^{5}$.

Locoregional recurrence, cervical metastatic spread and chemoresistance largely account for therapeutic failure and cancer-related death in $\mathrm{HNSCC}^{6}$. These aggressive events have been revealed to be intricately associated with a unique cell subpopulation in bulk cancer, termed cancer stem cells (CSCs) or tumor-initiating cells (TICs) with potent self-renewal and tumor-seeding properties. These cells sustain tumor overgrowth and drive recurrence and metastatic dissemination ${ }^{7,8}$. Moreover, these unique characteristics have enabled CSCs as attractive and yet challenging therapeutic targets as evidenced by the facts that targeting key regulators or molecular pathways responsible for CSCs properties has yielded remarkable anti-cancer effects with promising translational potentials ${ }^{9}$. Previous pioneering work has documented several surface or functional markers for HNSCC CSCs including CD44, CD133, Bmi1, SOX2 and ALDH1 ${ }^{10-12}$. Particularly, SOX2 (Sex determining region Y box 2), the key transcriptional factor preferentially expressed in embryonic and adult stem cells, has been demonstrated as an essential marker and regulator underlying these unique properties of CSCs from diverse cancer origins ${ }^{13-15}$. Previous reports have documented some essential clues to support SOX2 as a key CSC regulator in $\mathrm{HNSCC}^{16-19}$. However, the detailed mechanisms concerning how SOX2 itself is regulated in HNSCC remain incompletely known.

The Hippo signaling pathway has increasingly been recognized as a pivotal and indispensable mediator in tissue homeostasis, regeneration and tumorigenesis ${ }^{20}$. Dysregulation of Hippo signaling essentially contributes to cancer initiation, outgrowth, metastatic dissemination and therapeutic resistance. Upon Hippo inactivation, two downstream effectors transcriptional coactivator PDZbinding motif (TAZ/WWTR1) and yes-associated protein (YAP) are translocated into nucleus whereby they drive transcription of target genes mainly by forming complexes with TEA domain DNA-binding family of transcription factors (TEADs $)^{21}$. Importantly, we and others have reported that elevated TAZ promotes self-renewal and tumor-seeding potentials of CSCs and also confers CSCslike properties on differentiated non-CSCs in diverse cancer contexts ${ }^{22-24}$. However, the accurate downstream targets responsible for TAZ in HNSCC CSCs self-renewal and maintenance remain underexplored yet.

Here, we sought to determine whether SOX2 was a novel downstream target of TAZ underlying CSCs properties in HNSCC. Our findings by integrating cellular experiments in vitro, tumor-forming assay in xenograft animal model as well as bioinformatics data mining provide evidence that TAZ enhances CSCs self-renewal and maintenance by direct transcriptional activation of SOX2 in HNSCC.

\section{Results \\ SOX2 is a potential downstream target of TAZ in HNSCC}

Growing evidence has suggested that TAZ facilitates CSCs maintenance, self-renewal and expansion in multiple cancer contexts including HNSCC ${ }^{22,23,25}$. However, the accurate downstream targets responsible for TAZ underlying HNSCC CSCs properties remain largely underexplored. To address this, we set out to perform initial screen of dozens of putative CSCs regulators such as CD44, Nanog, SOX2, Bmi1 and ALDH1 as potential candidates via shRNA-mediated TAZ knockdown approach in vitro (data not shown). Among these candidates screened, SOX2 attracted our attentions due to the following reasons: its consistent and significant reduction upon TAZ silencing in both Cal27 and Fadu cells, its wellestablished roles in CSCs in squamous cell carcinoma as well as its intricate link with Hippo pathway in other biological contexts ${ }^{11,15,17,26}$. TAZ knockdown mediated by shRNA lentiviral constructs remarkably reduced both mRNA and protein abundance of SOX2, as well as CTGF (a well-known downstream target of TAZ) in vitro (Fig. 1a). In contrast, SOX2 was markedly upregulated in TAZ-overexpressing HNSCC cell lines (Fig. 1b). Complementarily, given TEADs as key transcriptional coactivators and mediators for TAZ functions and TEAD4 as a pivotal pro-oncogenic regulator in $\mathrm{HNSCC}^{21,27}$, TEAD4 knockdown via two independent siRNA oligonucleotides resulted in significant reduction of SOX2 expression in Cal27 and Fadu cells (Fig. 1c). Moreover, we employed online bioinformatics platform JASPAR (http://jaspar. genereg.net/) to predict potential binding sites of TEAD4 in human SOX2 promoter region (2000 bp upstream of TSS and $150 \mathrm{bp}$ downstream of TSS) and identified eight potential binding sites (Fig. 1d), thus supporting the potential regulation of SOX2 by TEAD4. On the other side, two reports have revealed that SOX2 regulates YAP expression directly or indirectly by modulating Hippo signaling in diverse biological settings ${ }^{26,28}$. However, we introduced SOX2 into cells and failed to observe significant changes of TAZ protein and its phosphorylation upon SOX2 overexpression in $293 \mathrm{~T}$, Fadu and HN6 cells (Fig. S1a-e). Moreover, the mRNA abundance of TAZ was not significantly affected by SOX2 overexpression as measured by qRT-PCR (Fig. S1b-f). Thus, our data suggested that TAZ might be not directly regulated by SOX2 in HNSCC. Collectively, these findings suggested that SOX2 might serve as a novel and important downstream target and mediator of TAZ during HNSCC tumorigenesis. 


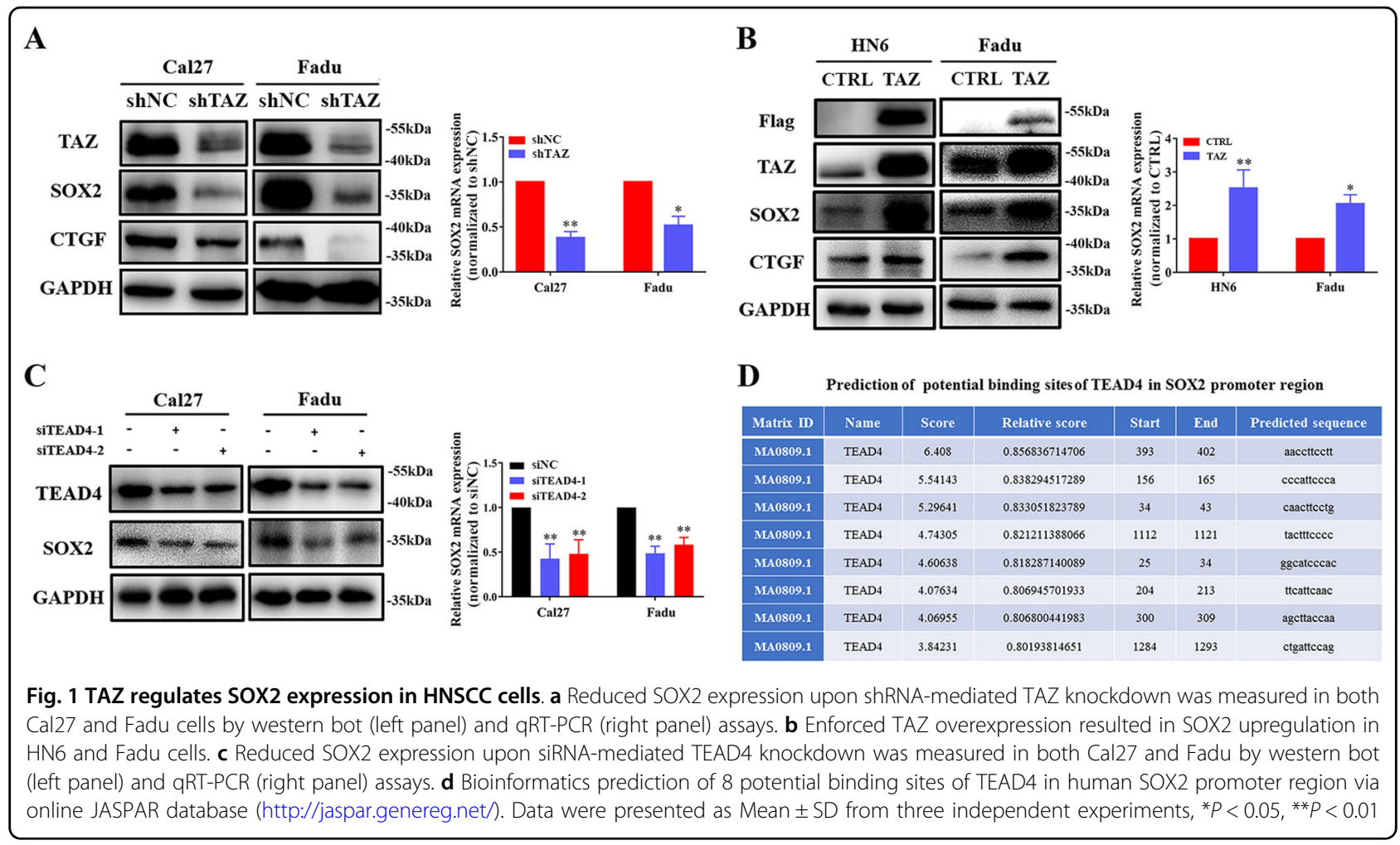

\section{SOX2 promotes CSCs stemness in HNSCC}

Given the essential roles of SOX2 in normal stem cells and malignant CSCs, we next sought to determine whether it was capable to modulate the properties of CSCs in HNSCC. We have previously identified a unique subpopulation with CSCs properties in HNSCC using surface markers CD44 and $\mathrm{CD} 133\left(\mathrm{CD} 44^{+} \mathrm{CD} 133^{+}\right.$defined as $\left.\mathrm{CSCs}\right)$ and demonstrated its robust self-renewal and tumor-initiating potentials both in vitro and in vivo ${ }^{23}$. As shown in Fig. S2, $\mathrm{CD} 44^{+} \mathrm{CD} 133^{+}$CSCs subpopulation yielded much more and larger tumorsphere in vivo as compared to $\mathrm{CD} 44^{-} \mathrm{CD} 133^{-}$subpopulation (non-CSCs). Then we utilized two independent siRNAs targeting human SOX2 to knockdown its expression and subsequently monitored the resulting phenotypic changes in vitro (Fig. 2a). In agreement with previous reports regarding SOX2 roles in cancer cell proliferation and apoptosis ${ }^{18,29}$, SOX2 knockdown by siRNA resulted in significant anti-proliferative and apoptosisinducing effects in vitro as measured by CCK- 8 , BrdU incorporation and Annexin V-PI flow cytometric assays (Fig. S3). As shown in Fig. 2b, c, SOX2 depletion resulted in significant downregulation of several CSCs markers and mediators such as CD44, CD133 and ALDH1A1. Meanwhile, the potentials of tumorsphere formation were remarkably impaired after SOX2 depletion (Fig. 2d, e). In addition, following separation of $\mathrm{CD} 44^{+} \mathrm{CD} 133^{+} \mathrm{CSCs}$ and CD $44^{-} \mathrm{CD}_{133^{-}}$non-CSCs by FACS from Cal27 and Fadu cells, significant enrichments of TAZ, SOX2, OCT4 and
Bmi1 protein expression were found in $\mathrm{CD} 44^{+} \mathrm{CD} 133^{+}$ CSCs as compared to $\mathrm{CD} 44^{-} \mathrm{CD} 133^{-}$non-CSCs (Fig. 2f). Next, we conducted serum-induced in vitro differentiation of tumorsphere assay and determined the expression changes of TAZ and SOX2 during this process. As shown in Fig. 2g, h, when tumorsphere was cultured and then induced to differentiation with serum-containing media, these floating tumorsphere gradually attached the plates and grown into monolayers within 3 to 7 days. Accompanying this, SOX2 and TAZ protein expression were remarkably reduced in serum-induced differentiation cells (Day 7) as compared to tumorsphere (Day 1). More importantly, we utilized limited dilution and tumorigenic assay in vivo to further determine whether SOX2 was required for selfrenewal and tumorigenesis in HNSCC. When stable SOX2knockdown cells by shRNA lentiviral constructs were selected and transplanted subcutaneously with diverse amounts of cells, much lower incidence of tumor formation was observed in SOX2-depleted cells (Fig. 2i). Collectively, these findings together with others provide compelling evidence that SOX2 is critically involved in HNSCC initiation and progression at least in part by modulating CSCs stemness $^{17-19}$.

\section{SOX2 is a key downstream pro-tumorigenic mediator of TAZ in HNSCC}

To further reinforce the notion that SOX2 is a novel downstream mediator of TAZ in HNSCC tumorigenesis, 


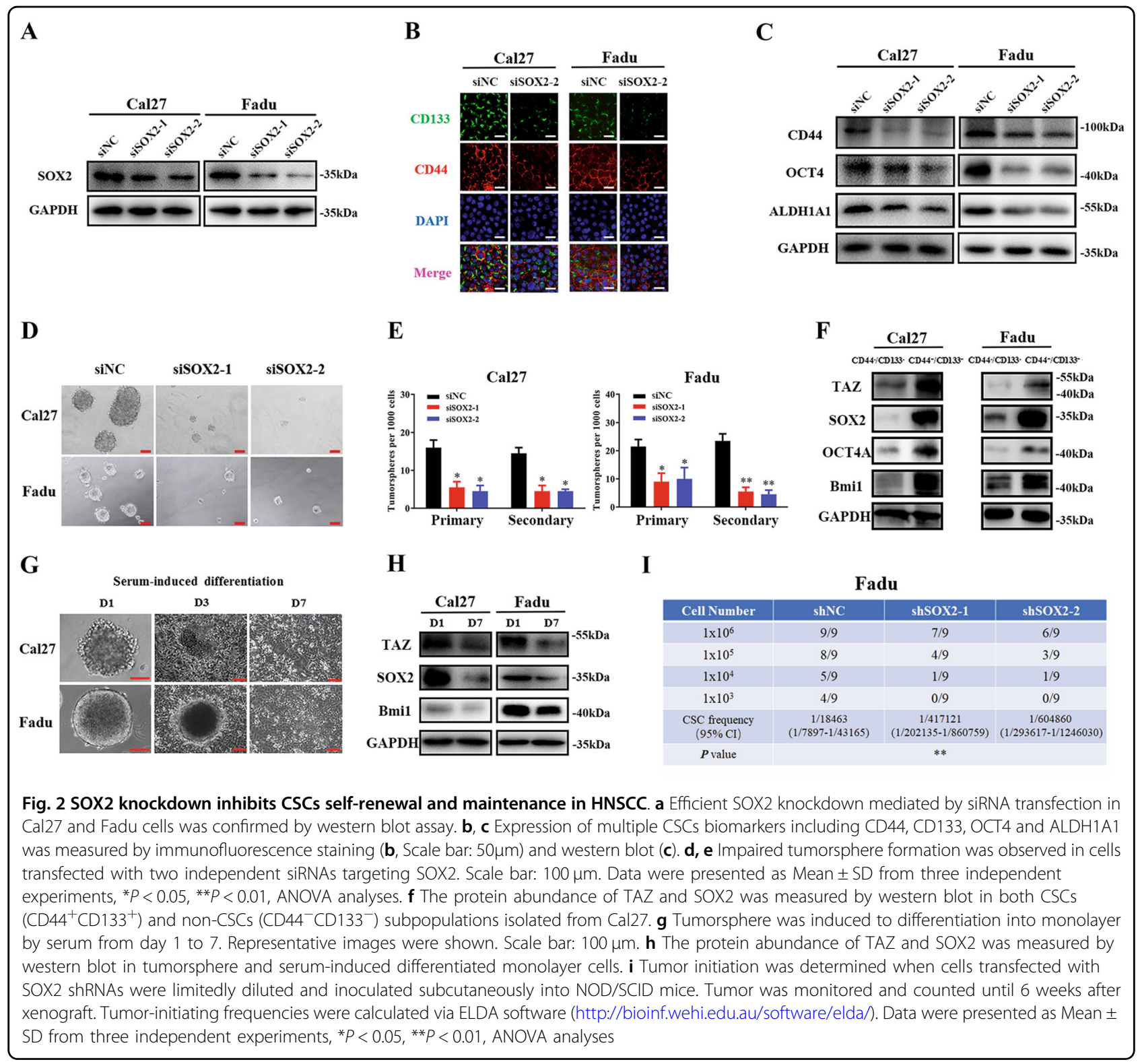

especially in the CSCs maintenance, we next performed rescue experiments and tumor-initiating assay via in vivo xenograft animal model. As shown in Fig. 3a, consistent with previous reports ${ }^{23}$, TAZ knockdown resulted in significant reduction of several CSCs markers like CD44, CD133 and ALDH1 in Fadu cells. Enforced overexpression of SOX2 by lentiviral constructs induced marked increase of these markers in Fadu cells (Fig. 3b). Notably, enforced overexpression of SOX2 largely rescued the expression of these CSCs markers in Fadu cells with stable TAZ knockdown (Fig. 3c). Additionally, as shown in Fig. 3d, e, our results from CCK-8 assays revealed that enforced overexpression of SOX2 largely abrogated the anti-proliferative effects as well as cisplatin sensitivity following TAZ depletion. Moreover, our data from transwell invasion and tumorsphere formation assays further indicated that reintroduction of SOX2 had the abilities to reverse, at least in part, the impaired invasiveness and self-renewal induced by TAZ depletion in vitro (Fig. 3f, g).

To recapitulate these in vitro findings in vivo, we utilized the limited dilution and tumorigenic assay. Various amounts of cells $\left(10^{3}-10^{6}\right.$ cells) with stable TAZ knockdown, SOX2 overexpression and TAZ knockdown plus SOX2 overexpression were subcutaneously inoculated into both blanks of NOD/SCID mice. Consistently, as shown in Fig. 4a, TAZ depletion significantly impaired tumor-initiating potentials, while SOX2 overexpression enhanced tumor-initiating properties in vivo. Noticeably, when SOX2 was reintroduced into cells with stable TAZ 


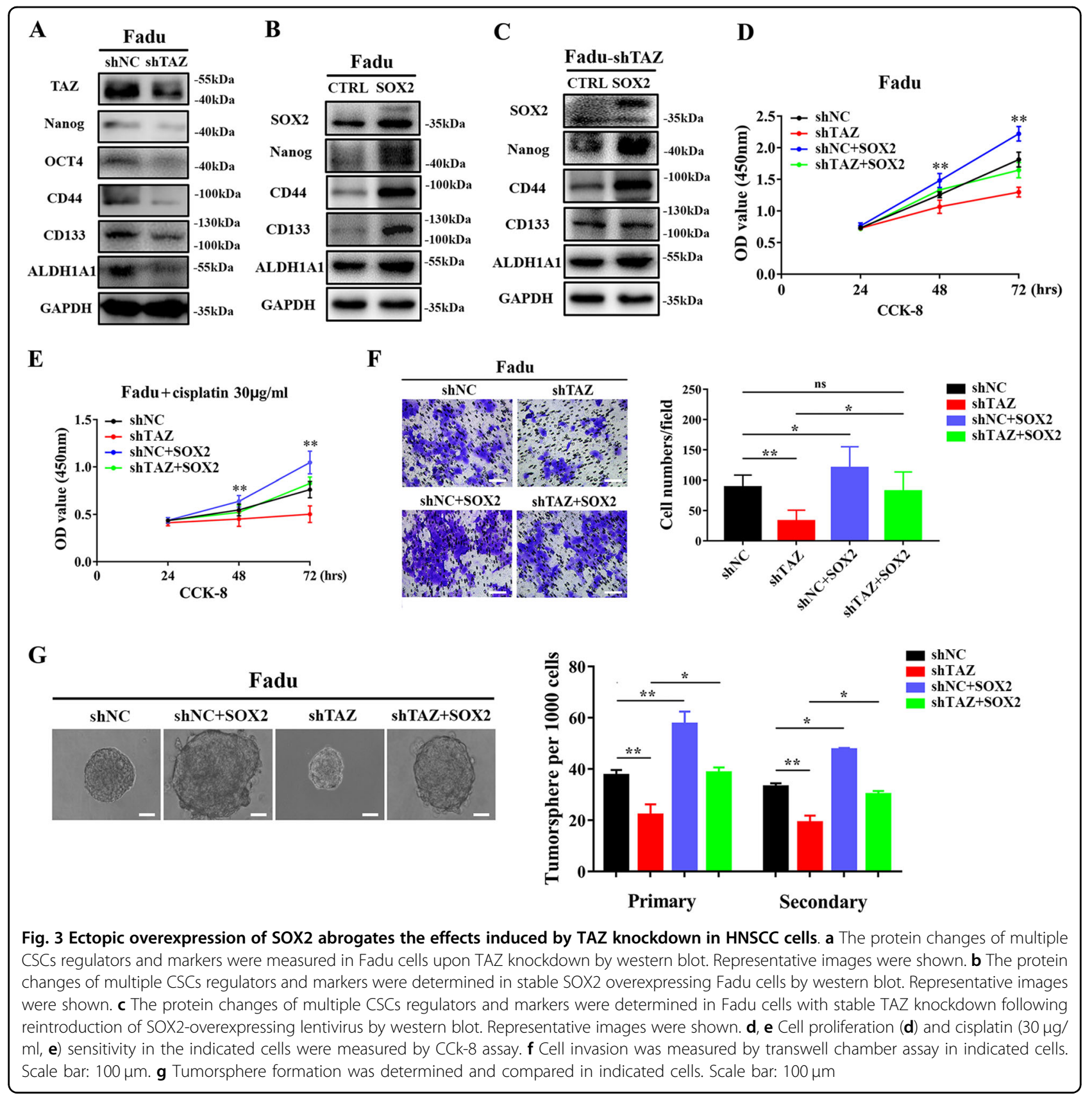

knockdown, this manipulation was capable to partially restore their tumor-forming properties in vivo. As displayed in Fig. 4b-e, these samples were formed from transplantation with $10^{6}$ cells within 4 weeks. Data from volume during tumor growth, final volume and weight of tumor samples indicated that TAZ and SOX2 facilitated tumor overgrowth in vivo. Notably, enforced SOX2 overexpression was capable to partially abrogate the antigrowth effects resulted from TAZ knockdown. Then, tumor samples derived from Fadu cells with TAZ or/and SOX2 manipulations were subjected to further analyses.
As shown in Fig. 4f, the expression levels of ALDH1A1, CD44 and CD133 were restored in samples derived from TAZ knockdown plus SOX2-overexpressing cells as compared to those from TAZ knockdown cells. Concomitantly, immunohistochemical staining coupled with quantitative analyses further supported that SOX2 overexpression had capacities to restore the expression abundance of these CSCs markers in HNSCC (Fig. 4g-k). Taken together, our findings strongly support that SOX2 is an important downstream mediator of TAZ in regulating CSCs selfrenewal and tumorigenic potentials in HNSCC. 


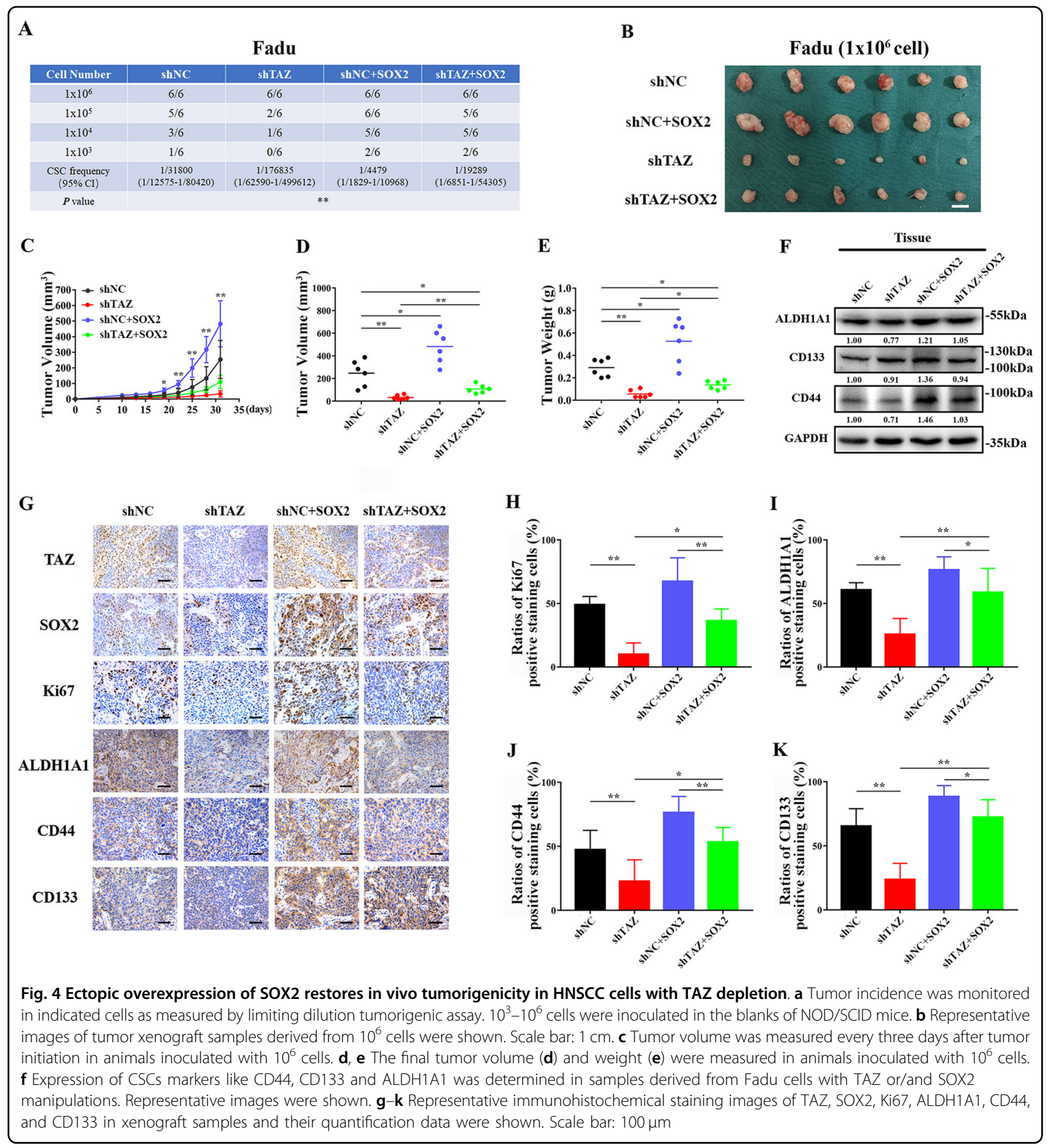

TAZ-TEAD4 complex directly activates SOX2 transcription in HNSCC

Previous reports have demonstrated that Hippo effectors TAZ and YAP primarily bind with TEADs and act in concert to dictate downstream transcriptional outputs during diverse biological and pathological processes ${ }^{20,21}$. Here, we selected TEAD4 as the TAZ primary binding partner from four TEAD members due to its significant and consistent upregulation and key roles in HNSCC as we reported before ${ }^{27}$. Thus, we performed protein immunoprecipitation (IP) experiments in Cal27 and Fadu cells and found endogenous TEAD4 in complex immunoprecipitated by TAZ specific antibody (Fig. 5a). Next, we cloned the sequence of human SOX2 promoter by PCR and generated wide-type luciferase reporter of SOX2 promoter (WT, SOX2-luc) and conducted luciferase 


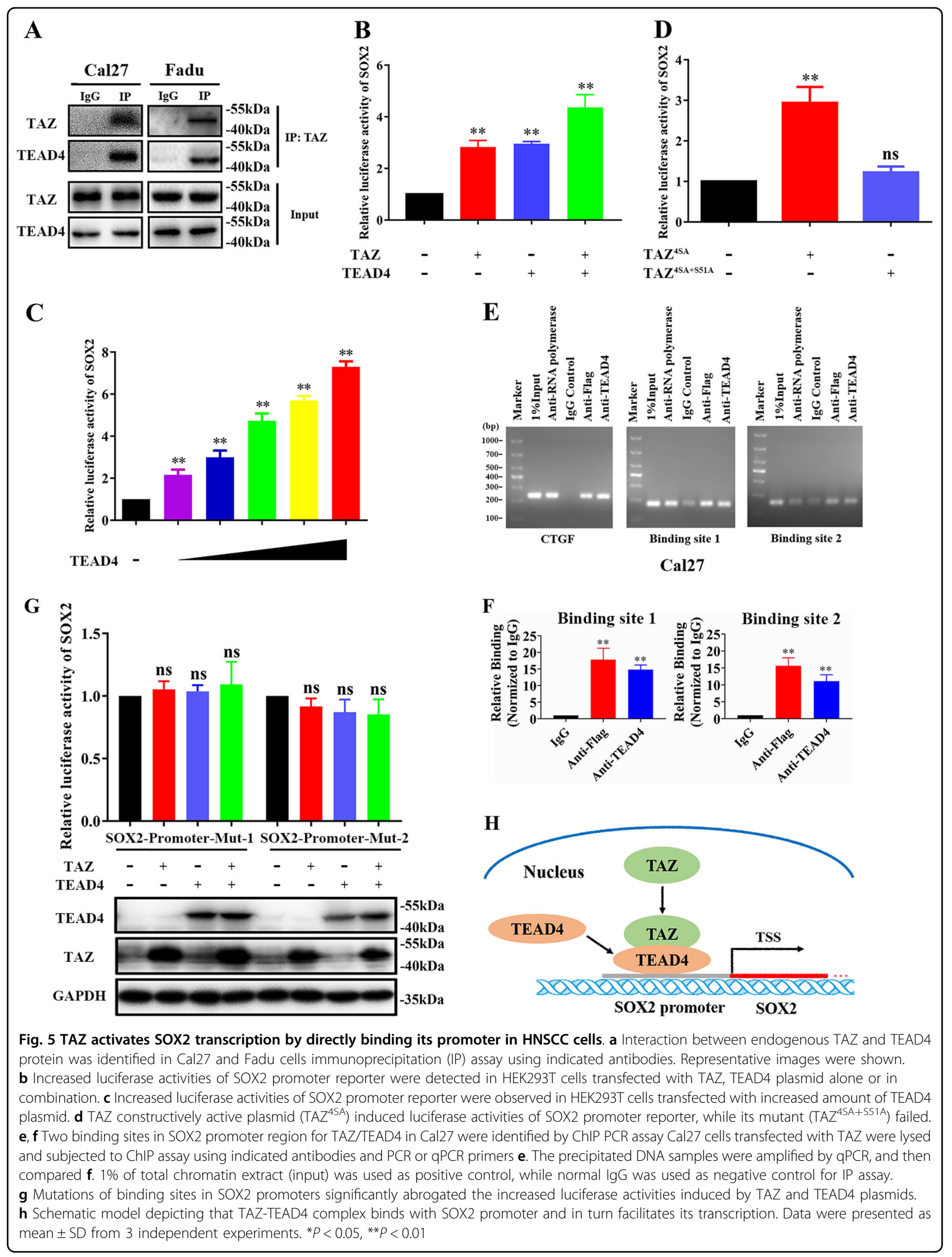


reporter assays. This reporter vector was simultaneously transfected with TEAD4-overexpressing or/and TAZoverexpressing constructs into HEK293T. Significantly increased luciferase activities were observed in cells cotransfected with TEAD4 or TAZ overexpressing plasmids. As expected, much more luciferase activities were detected in cells transfected with both TAZ and TEAD4 vectors (Fig. 5b). Additionally, transfection with increased amount of TEAD4 constructs significantly enhanced luciferase activities in a dose-dependent manner (Fig. 5c). Moreover, TAZ mutant construct $\left(\mathrm{TAZ}^{4 \mathrm{SA}+\mathrm{S} 51 \mathrm{~A}}\right)$ without TEAD4 binding ability failed to increase luciferase activities of SOX2 promoter (Fig. 5d). Furthermore, we designed individual primers spanning 8 potential binding sites in SOX2 promoter (as shown in Fig. 1d), performed ChIP-PCR assay and found significant enrichments of TEAD4 binding in two sites $(-309$ to $-300,-1292$ to -1285 , Fig. 5e). Consistently, we also designed specific primers, performed ChIP-qPCR assay and revealed that both TAZ and TEAD4 were significantly enriched in these two binding sites in SOX2 promoter (Fig. 5f). Furthermore, we generated two mutant luciferase reporters (SOX2-mut-1/2) by site-directed mutagenesis in these two sites in SOX2-luc WT plasmid. As shown in Fig. 5g, transfections of TAZ, TEAD4 overexpressing plasmid alone or in combination failed to induce significant effects on luciferase activities in cells co-transfected with SOX2 mutant promoter reporters. Taken together, as schematic illustration in Fig. 5h, our results indicate that TAZTEAD4 complex directly binds with SOX2 promoter and in turn facilitates its transcription in HNSCC.

\section{Prognostic significance of TAZ and SOX2 co-expression in HNSCC}

Having documented important roles of TAZ/TEAD4SOX2 regulatory axis during HNSCC tumorigenesis, we next sought to determine whether their expression had prognostic significance in patients with primary HNSCC. The mRNA expression of TAZ and SOX2 in 73 primary HNSCC samples and paired adjacent non-tumor epithelial was measured by qRT-PCR assay. In addition, the protein levels of TAZ and SOX2 in 12 paired freshly collected HNSCC and adjacent non-tumor epithelial were also detected by western blot. As shown in Fig. 6a-d, significant upregulations of TAZ and SOX2 at both mRNA and protein levels were detected in HNSCC samples relative to their non-tumor counterparts. Moreover, positive correlations were found between TAZ and SOX2 at both mRNA and protein levels in these samples examined (Fig. 6c, e, Person correlation). Consistently, we mined the transcriptional profiling data of HNSCC samples from TCGA and GEO datasets and also found positive correlations between TAZ mRNA and SOX2 mRNA from three independent patient cohorts (Fig. S4).
Next, we further determined the abundance of TAZ and SOX2 by immunohistochemical staining in archived HNSCC samples with detailed follow-up data available. As shown in Fig. 6f, obvious nuclear staining of TAZ and SOX2 was detected in cancerous cells while much less staining of SOX2 or cytoplasmic staining of TAZ was identified in non-tumor oral epithelial cells. According to our immunohistochemical scoring regime, as listed in Table S5, high TAZ expression was found in 36 patients while elevated SOX2 expression was detected in 30 patients. Significant associations were found between TAZ/SOX2 abundance and cervical nodal metastasis, as well as tumor size (Chi-square test, $P<0.05$ ), while no significant correlations between TAZ/SOX2 and other clinicopathological parameters were identified. In addition, significant correlation between TAZ and SOX2 expression was found in these samples examined (Fig. 6g). Next, we applied Kaplan-Meier analyses to determine the prognostic significance of TAZ/SOX2 co-expression in HNSCC and revealed that patients with $\mathrm{TAZ}^{\text {high }} \mathrm{SOX} 2^{\text {high }}$ expression had the worst prognosis as evidenced by the lowest overall survival among four subgroups categorized by TAZ and SOX2 expression (Fig. 6h). To further extend these findings, we exploited a bioinformatics approach by identification and filtering the overlapped candidates which were significantly correlated with TAZ or SOX2 in TCGA-HNSCC dataset. As shown in Fig. 7a, a total of 1312 overlapped genes were initially found between TAZ or SOX2- correlated genes in TCGA-HNSCC dataset. These genes were significantly enriched in diverse signaling pathways critically involved in CSCs self-renewal such as Wnt, Hedgehog and Notch pathways (Fig. 7b), and functional categories such as somatic stem cell division (Fig. 7c). Finally, to determine whether these TAZ/ SOX2-correlated genes had prognostic significance in HNSCC, we developed a prognostic score comprising 5 genes (UBN2, DUSP16, DSG2, FXR1 and SC5D) by sequential univariate regression analysis, Robust likelihood-based modeling and multivariate regression analysis using TCGA-HNSCC dataset as training cohort (Fig. 7d). This risk score was calculated by the following formula: risk score $=(-0.39864 \times \mathrm{UBN} 2)+(-0.31743 \times$ DUSP16 $)+(0.18477 \times$ DSG2 $)+(0.39579 \times$ FXR1 $)+$ $(0.28306 \times$ SC5D). The optimal cutoff for this score was 1.141 derived from the ROC curve using TCGA-HNSCC dataset (Fig. 7e). Then Kaplan-Meier analyses indicated that patients with high scores had markedly reduced survival as compared to those with low scores in TCGAHNSCC dataset $(P<0.0001$, Fig. $7 \mathrm{f})$. Data from another two independent cohorts of HNSCC samples (GSE41613 and GSE42743) were further utilized as the testing and validation cohorts to verify the prognostic utility of this score. Consistent with the findings from the training set, patients with high scores had significantly lower OS ratios 


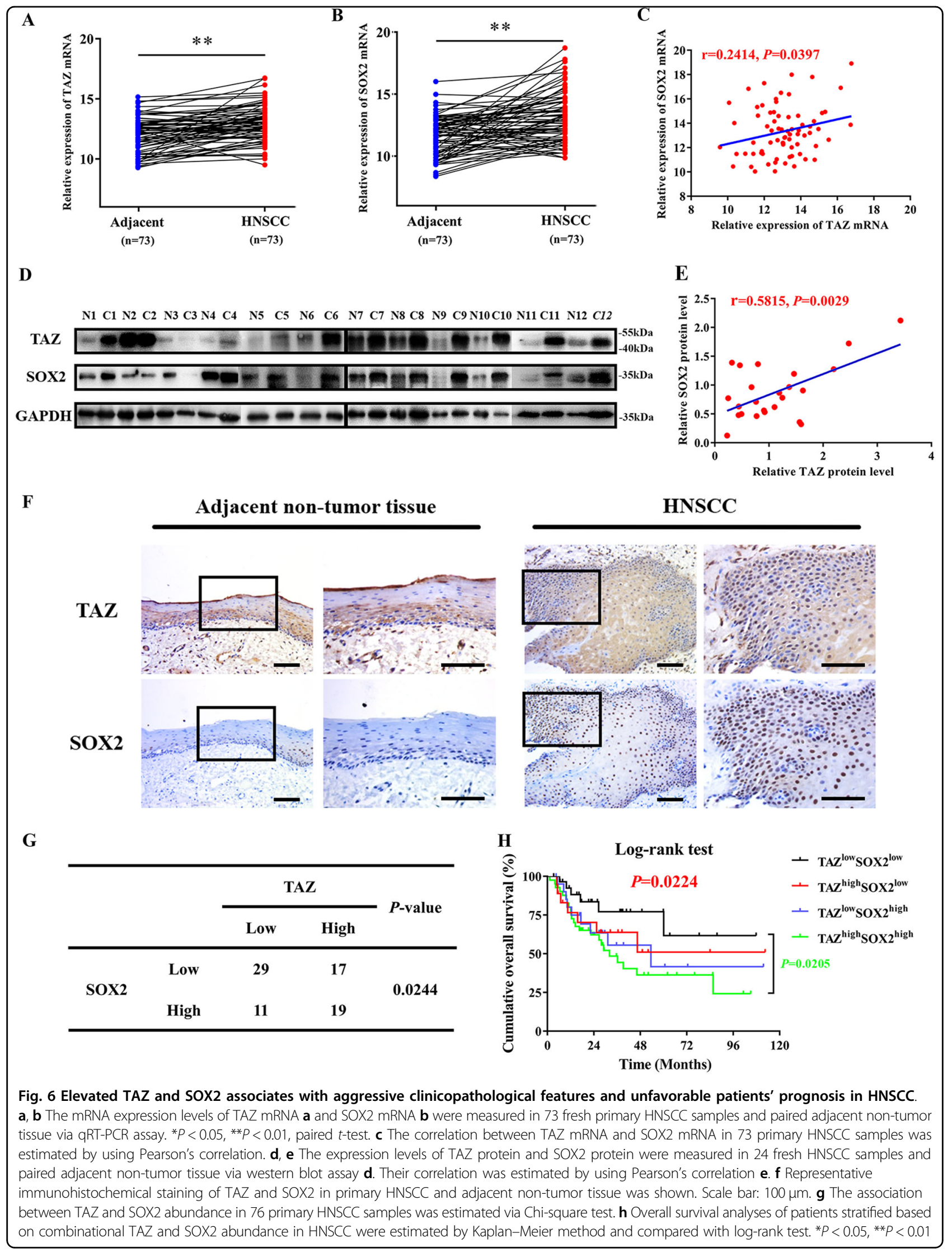




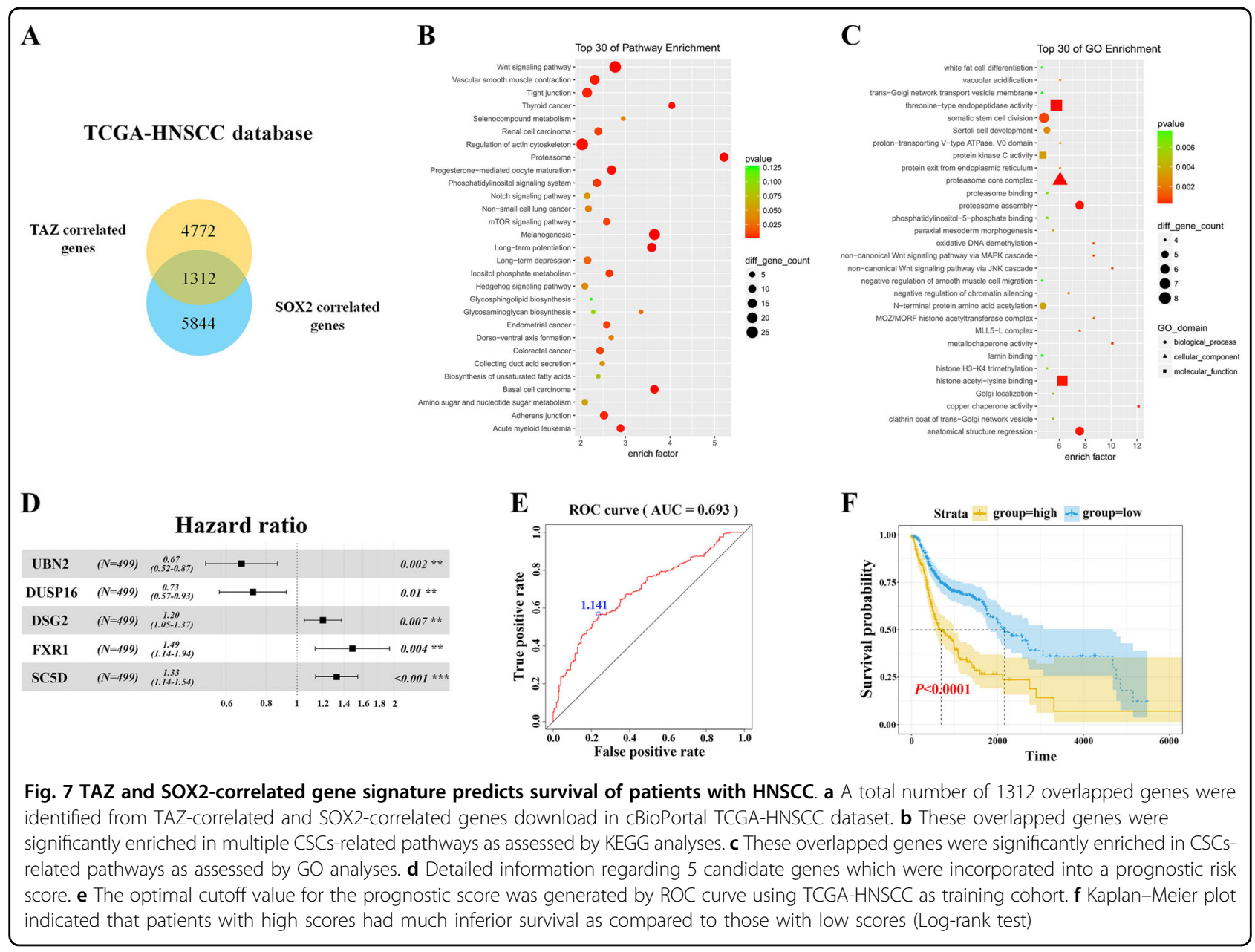

as compared to those with low scores in both testing and validation cohorts with favorable sensitivity and specificity $(P=0.00054,0.00028$; Log-rank test; Fig. S5). To reinforce the prognostic value of this score and rule out other confounding factors, we performed univariate and multivariate cox regression analyses and found that this score was an independent prognostic predictor for overall survival in the TCGA-HNSCC cohort (Table S5, Table S6). Taken together, our data indicate that TAZ/TEAD4SOX2 axis associates with aggressive clinicopathological features and unfavorable patients' prognosis in HNSCC.

\section{Discussion}

Similar with other malignancies, HNSCC is characterized by a histologically heterogeneous population of cancer cells as evidenced that very small fraction of cells serves as CSCs or tumor-initiating cells that are critical for cancer initiation, recurrence, metastatic spreading and therapeutic resistance ${ }^{9,10,12}$. Dysfunctional Hippo signaling pathway has been increasingly recognized as a central mediator for tumorigenesis, as well as a potential therapeutic target in diverse cancer contexts ${ }^{21}$. In particular,
TAZ has been identified as an essential modulator for CSCs self-renewal and maintenance in several human malignancies ${ }^{22,23}$. However, the precise downstream targets of TAZ and relevant underlying mechanisms for its function in HNSCC CSCs remain incomplete known. Here, our findings reveal that TAZ activates SOX2 transcription by directly binding to its promoter region and in turn facilitates CSCs maintenance and tumorigenicity in HNSCC.

Our previous results have showed that TAZ mediates self-renewal and maintenance of CSC in OSCC as evidenced by its enrichment in CSC subpopulation, impaired tumorsphere formation and reduced CSC percentage upon TAZ knockdown, as well as positive associations between TAZ expression and tumor aggressiveness in OSCC samples ${ }^{23,30}$. Moreover, enforced TAZ overexpression endowed non-CSCs with CSCs-related properties presumably by inducing epithelial-mesenchymal transition (EMT) in breast cancer and $\mathrm{OSCC}^{22,23}$. To extend these findings and bridge the gap between TAZ and CSCs stemness in HNSCC, we screened dozes of known CSCs regulators and found that SOX2 might be 
downstream target of TAZ in HNSCC as evidenced by its downregulation or upregulation followed by TAZ knockdown or overexpression in vitro. Consistent with previous findings regarding SOX2 in HNSCC stemness $^{16,18,19,31}$, our data further confirmed the pivotal roles of SOX2 in CSCs maintenance in HNSCC as evidenced by impaired abilities of tumorsphere formation, reduced expression of CSCs markers and compromised tumor initiation in vivo following SOX2 knockdown. Additionally, both TAZ and SOX2 were significantly enriched in CSCs subpopulation and were significantly downregulated during serum-induced tumorsphere differentiation. More importantly, experimental findings derived from limiting dilution and tumorigenic assay in vivo further revealed the pivotal roles of TAZ and SOX2 required for HNSCC tumor initiation and overgrowth. Collectively, these findings provide compelling evidence to support the essential roles of TAZ and SOX2 in CSCs self-renewal and maintenance in HNSCC.

Previous studies have established that TAZ primarily functions as a transcriptional coactivator in complex with TEADs to mediate downstream transcriptional events $^{21,32}$. Disruption of TAZ-TEADs binding blocked the major effects mediated by TAZ including oncogenic transformation, pro-proliferation and EMT in cancer ${ }^{33}$. Here, we identified TEAD4 as the key partner with TAZ to regulate SOX2 expression in HNSCC through IP assay, which was also consistent with its oncogenic functions and aberrant upregulation in cancer ${ }^{27,34}$. Subsequently, our results from luciferase reporter assays involving SOX2 promoter and its mutant as well as ChIP assays further confirmed the direct binding between TAZ/TEAD4 and SOX2 promoter in HNSCC. Noticeably, TAED binding sites in SOX2 promoter identified here were different from those found in other reports whereby two putative YAP/TEAD binding sites were found at upstream $(-3759)$ and downstream $(+5313)$ of SOX2 transcription start site (TSS) in murine osteosarcoma cells ${ }^{35}$. We reasoned that it's conceivable that binding sites for transcriptional factors vary in diverse cell types and biological settings. More importantly, reintroduction of SOX2 into cells with TAZ stable knockdown abrogated, at least in part, the impaired proliferation, migration and tumorsphere formation, and in vivo tumor initiation and growth induced by TAZ depletion. Interestingly, several previous reports revealed that YAP1 transcriptionally activated SOX2 expression through a physical interaction with OCT4 to facilitate self-renewal of stem-like cells in lung cancer $^{28}$. SOX2 antagonized Hippo pathway leading to exaggerated YAP functions to maintain stemness in osteosarcoma ${ }^{15}$. However, our data failed to support TAZ as a potential, direct downstream target of SOX2, which was consistent with previous report wherein TAZ was unaffected in SOX2-depleted osteoprogenitors ${ }^{26}$. Taken together, these in vitro and in vivo findings offer experimental support that TAZ-TEAD4 complex activates SOX2 transcription by direct binding with its promoter, which in turn modulates CSCs stemness in HNSCC. These findings identified another upstream regulator of SOX2 and added another layer of complexity to the transcriptional regulatory network governing CSCs unique characteristics.

The prognostic value of TAZ has been established in several human cancer including OSCC and HNSCC $^{23,25,30}$. However, the expression pattern of SOX2 and its prognostic significance in HNSCC remain inconsistent and contradictory. Some reports found that SOX2 was frequently amplified in HNSCC and its upregulation associated with favorable survival, while others documented the opposite results ${ }^{36-40}$. In our patient cohort, we failed to identify positive or negative association between SOX2 expression and patient survival (data not shown). These discrepancies might be due to sample size, tumor heterogeneity as well as methods of patient stratification. Nonetheless, our results revealed that patients with $\mathrm{TAZ}^{\text {high }} / \mathrm{SOX} 2^{\text {high }}$ had the worst survival ratios as compared to other patient subgroups. Of course, further studies are needed to resolve the inconsistency regarding the prognostic value of SOX2 in multiple, large and independent HNSCC cohorts. Noticeably, we developed a prognostic score based on TAZ/SOX2-correlated genes via bioinformatics and statistical approaches and found that this score had potent power to stratify HNSCC patients into subgroups with favorable or inferior survival. This suggests that the regulatory network modulated by TAZ and SOX2 in HNSCC might not only intricately contribute to tumorigenesis, but also might be exploited as a novel prognostic biomarker with translational promise in the clinic.

In conclusion, our data reveal a previously unknown mechanistic linkage between TAZ and SOX2 and identify SOX2 as a direct downstream target of TAZ in modulating CSCs self-renewal and maintenance in HNSCC. These findings suggest a novel therapeutic approach to target TAZ/TEAD4-SOX2 signaling axis in HNSCC.

\section{Materials and methods \\ Cell culture and regents}

A panel of HNSCC cell lines including Cal27, Fadu, HN6 and human embryonic kidney 293 T (HEK293T) was used here. Cal27, Fadu and HEK293T were obtained from American Type Culture Collection (ATCC, Manassas, VA, USA) and authenticated by short tandem repeat profiling at regular intervals. HN6 was a generous gift from Prof. Wantao Chen (Shanghai Jiaotong University). All cancerous cells lines were maintained in DMEM/F12 (Gibco) supplemented with $10 \%$ fetal bovine serum (Gibco) and $1 \%$ penicillin/streptomycin at $37^{\circ} \mathrm{C}$ in $5 \%$ 
$\mathrm{CO}_{2}$. Mycoplasma detection was routinely performed during the whole course of this study. All regents were purchased from Sigma-Aldrich unless otherwise stated.

\section{Small interference or hairpin RNA, DNA constructs, viral production and transfection/infection}

Two independent sequences of siRNA or shRNA targeting human SOX2 and TEAD4 mRNA (detailed sequences were listed in Table S1) were designed and synthesized from GenePharma company (Shanghai, China). These siRNAs were transiently transfected into cells with lipofectamine 2000 (Invitrogen) at final concentration of $100 \mathrm{nM}$ unless otherwise specified. Two short hairpin RNAs (shRNAs) against human TAZ mRNA or TAZ overexpression lentiviral construct tagged with single N-Flag was generated as we previously reported $^{23}$. The TAZ mutant plasmids $\left(\mathrm{TAZ}^{4 \mathrm{SA}}\right.$ and $\mathrm{TAZ}^{4 \mathrm{SA}+\mathrm{S} 51 \mathrm{~A}}$ ) were kindly gifted from Prof. Kunliang Guan $^{41}$. The human full-length SOX2 or TEAD4 cDNA with $3 \times$ Flag was subcloned into lentiviral plasmid pLenti $\mathrm{CMV} /$ Puro and then verified by direct sequencing. Lentiviral particles were prepared by transiently cotransfecting HEK293T cells with individual lentiviral constructs and controls together with packaging and envelope plasmids (pCMV-VSV-G and pCMV- 48.2 ) using the calcium-phosphate method. These viral supernatants were filtered, concentrated and stored until use. For transient transfection assay with siRNA or plasmids, cells were harvested at $48 \mathrm{~h}$ for further experiments. To gain stable clones after infections with shRNA or overexpression lentiviral vectors, cells were selected with puromycin $(2-5 \mu \mathrm{g} / \mathrm{ml}$, Sigma) for at least one week.

\section{RNA extraction, and quantitative real-time PCR (qRT-PCR)}

Total RNA of tissue specimens or cells was extracted with Trizol reagent (Invitrogen) and then subjected to transcription into cDNA by PrimeScript ${ }^{\mathrm{Tm}} \mathrm{RT}$ Master Mix (Takara) according to the manufacturer's instructions. PrimeScript ${ }^{\mathrm{TM}}$ RT-PCR kit (Takara) was used for qRT-PCR reactions, as we described previously ${ }^{23,42}$. Endogenous $18 \mathrm{~S}$ RNA or GAPDH was used for data normalization. All qPCR primers used were listed in Table S2.

\section{Cell viability, proliferation and invasion assay}

Cell proliferation and viability were assessed by absorbance using CCK-8 cell viability assay (Cell Counting Kit8, Dojindo, Japan) and BrdU incorporation assay according to manufacturer' instructions. $\mathrm{BrdU}^{+}$cells were identified under fluorescent microscopy, photographed and counted via ImageJ software. Cell invasion was assessed using transwell chambers with $8-\mu \mathrm{m}$ pore size (Corning) with pre-coated Matrigel (BD Pharmingen) as we described previously ${ }^{43}$.
Flow cytometry and fluorescence active cell sorting (FACS)

Flow cytometry for cell apoptosis and fluorescenceactivated cell sorting were similar as we reported previously $^{23}$. Briefly, for apoptosis detection, cells were trypsinized, dissociated into single cell suspension, then assayed with Annexin V: PE Apoptosis Detection Kit (BD Bioscience) for flow cytometry. For FACS, single cell suspension was incubated with CD44 (560890, BD Pharmingen, 1:100) and CD133/1 (AC133, Miltenyi, 1:100) and two subpopulations of $\mathrm{CD} 44^{+} \mathrm{CD} 133^{+}$and $\mathrm{CD} 44^{-} \mathrm{CD} 133^{-}$was separated when corresponding immunoglobulins was used for blank control. All data were collected and analyzed by BD FACSuite software.

\section{Western blot and immunoprecipitation (IP)}

Western blot analyses were routine performed as described previously ${ }^{23}$. GAPDH was used as a loading control. For co-IP assay, cells were lysed by the Western \& IP Lysate Buffer (Beyotime, China) supplemented with 1\% protease inhibitor (Roche) on ice for $>15 \mathrm{~min}$. Cell lysate was centrifuged under $4{ }^{\circ} \mathrm{C}$ for $10 \mathrm{~min}$ at $12,000 \mathrm{rpm}$, and the supernatant was incubated with primary antibodies and protein A/G agarose beads (Thermo Fisher Scientific) with rotating at $4{ }^{\circ} \mathrm{C}$ overnight. The pellet was washed at least three times with IP lysate buffer on ice and then subjected to western blotting analysis. All antibodies used were listed in Table S3.

\section{Luciferase reporter assay}

The promoter sequence (2065 bp) upstream of the transcriptional start site of human SOX2 was subcloned into a luciferase reporter plasmid and verified with direct sequencing. Two putative binding sites between TEAD4 and SOX2 were individually mutated using QuikChange ${ }^{\circledR}$ Lightning Site-Directed Mutagenesis Kits (Stratagene) and verified by direct sequencing. The $293 \mathrm{~T}$ cells were transiently transfected with pGL-SOX2, TAZ or TEAD4 plasmids and phRL-CMV plasmid (Promega) using lipofectamine 2000 (Invitrogen). Cells were harvested $24 \mathrm{~h}$ after transfection and assayed for Firefly and Renilla luciferase activity using the Dual-Luciferase reporter system (Promega). Data were presented as the ratios of Firefly to Renilla luciferase activity.

\section{Chromatin immunoprecipitation (ChIP) assay}

For ChIP assay, Cal27 cells that had been transfected with Flag-TAZ for $48 \mathrm{~h}$ were harvested and lysed using the EZ-ChIP ${ }^{\mathrm{TM}}$ Chromatin Immunoprecipitation Kit (Millipore) according to the manufacturer's instructions. Isolated and purified chromatin was incubated overnight with antibodies for TEAD4 (Abcam), Flag (Sigma), RNA polymerase (Millipore), or normal mouse IgG (Millipore). RNA polymerase was utilized as positive control and normal mouse IgG was used as negative control. The PCR 
or qPCR primers for detecting CTGF, SOX2 binding site 1 and 2 were listed in the Table S4.

\section{Tumorsphere formation assay}

The disassociated single cells $\left(10^{4} / \mathrm{ml}\right)$ were cultured in serum-free DMEM/F-12 supplemented with B27, $20 \mathrm{ng} / \mathrm{ml}$ EGF (BD Bioscience) and $10 \mathrm{ng} / \mathrm{ml} \mathrm{bFGF}$ (BD Bioscience) and grown in ultra-low-attachment plates (Corning) at routine conditions for 7-10 days. For in vitro serial passages, these tumorsphere were harvested and further dissociated into single cells by $0.1 \%$ trypsin and gentle pipette, and then filtered, re-plated to form secondary sphere in aforementioned medium. The tumorsphere with diameter larger than $50 \mu \mathrm{m}$ was counted.

\section{HNSCC xenograft animal model}

All animal models involved in this experiment were in accordance with the institutional animal welfare guidelines and protocols were approved by Institutional Animal Care and Use Committee of Nanjing Medical University. Six-week-old female NOD/SCID mice were purchased from Model Animal Research Center of Nanjing Medical University and maintained in the specific pathologic-free animal facility. Cancer cells were trypsinized, counted and diluted with equal number $\left(1 \times 10^{6}, 1 \times 10^{5}, 1 \times 10^{4}, 1 \times\right.$ $\left.10^{3}\right)$ in $200 \mu \mathrm{L}$ media $(100 \mu \mathrm{L}$ PBS plus $100 \mu \mathrm{L}$ PBS Matrigel) and then subcutaneously injected into both flanks of each animal (6 animals per experimental group). Tumor initiation and growth were monitored and recorded every three days. Tumor volume was calculated with the formula: volume $=a \times b^{2} / 2$. Tumor weight was weighed and recorded after animals sacrificed. Tissue samples were fixed in formalin or maintained in liquid nitrogen for further analyses.

\section{Patients and tissue specimens}

A total number of 73 fresh HNSCC samples and paired adjacent non-tumor epithelial tissues was obtained from patients between Jan. 2007 to Dec. 2015 at Department of oral and maxillofacial surgery, Affiliated Hospital of Stomatology, Nanjing Medical University. All samples were harvested within $30 \mathrm{~min}$ after surgical resection and histopathologically confirmed by senior pathologists. Seventy-six primary archived HSNCC samples from another independent patient cohort with detailed followup data were used for immunohistochemical staining. Patient inclusion criteria for these two cohorts were described as follows: primary HNSCC without any prior history of chemotherapy or radiotherapy; patients underwent radical tumor resection and elective or therapeutic neck dissection as required; detailed demographic, clinical and pathological data. Written informed consent was obtained from these patients or donors. This study protocol was reviewed and approved by the Research Ethic Committee of Nanjing Medical University.

\section{Immunohistochemical staining and scoring}

Immunohistochemical staining was routinely performed on $4 \mu \mathrm{m}$-thick sections from formalin-fixed paraffinembedded clinical samples as previously described. Negative controls (without primary antibody incubation) were included in each staining run. Immunoreactivity was semi-quantitatively evaluated according to staining intensity and distribution using the immunoreactive score which was calculated as intensity score $\times$ proportion score as we previously reported ${ }^{23,43,44}$. Intensity score was defined as 0 , negative; 1 , weak; 2 , moderate; 3 , strong. The proportion score was defined as 0 , negative; $1,<10 \% ; 2$, $11-50 \% ; 3,51-80 \% ; 4,>80 \%$ positive cells. The total score ranged from 0 to 12 . Accordingly, the immunoreactivity of each slide was categorized into three subgroups based on final scores: 0 , negative; 1-4, low expression; 4-12, high expression.

\section{Bioinformatics analyses of online transcriptional profiling data of HNSCC}

The original data concerning the expression of TAZ and SOX2 in HNSCC were retrieved from publicly available databases The Cancer Genome Atlas (TCGA, https:// cancergenome.nih.gov/) and Gene Expression Omnibus (GEO) Database (GSE23036 and GSE65858) ${ }^{45,46}$. The mRNA expression levels of TAZ and SOX2 HNSCC were log2-transformed and compared using the Pearson correlation test.

\section{Identification of TAZ/SOX2-correlated gene signature with prognostic value}

The positively or negatively correlated genes of TAZ and SOX2 in HNSCC were initially downloaded from cBioPortal (https://www.cbioportal.org/) using TCGAHNSCC dataset. The overlapped genes were considered as potential candidates for TAZ/SOX2-correlated gene signature, which were subjected to univariate regression assay to identify candidates which were significantly associated with overall survival (with $P$ value of less than $0.05)$ in TCGA-HNSCC datasets. Then, these candidates were further filtered using Robust likelihood-based modeling for 1000 times via $R$ environment with Rbsurv package and multivariate Cox regression analysis with top statistical significance ${ }^{44,47,48}$. A risk score formula based on the expression level and coefficient of these selected candidates was generated and its optimal cut-off point was selected at the maximal sensitivity and specificity by receiver operating characteristics (ROC) curve. Subsequently, the prognostic values of this score were validated in two publically available HNSCC cohorts (GSE41613 and GSE42743 ${ }^{49}$. 


\section{Statistical analysis}

All quantitative data in the present study were shown as mean \pm SD of three independent experiments unless otherwise stated. All data were analyzed using GraphPad Prism 7.0 or SPSS 22.0 software with Student's $t$-test and ANOVA with post-hoc test unless otherwise specified. The Chi-squared test was applied for TAZ and SOX2 expression and various clinicopathological parameters. The Kaplan-Meier method and Log-rank test were used for the assessment of patient survival. Differences were considered statistically significant at $P<0.05$ (*) and $P<$ $0.01{ }^{(* *)}$.

\section{Acknowledgements}

We thank Prof. Wantao Chen and Prof. Kunliang Guang for their kind gift of cell lines and plasmids. This work is financially supported, in whole or in part, by National Natural Science Foundation of China $(81572669,81602386$, 81602378), Natural Science Foundation of Jiangsu Province (BK20161564, BK20161024), A Project Funded by the Priority Academic Program Development of Jiangsu Higher Education Institutions (2018-87), Natural Scientific Research Project for College in Jiangsu Province (16KJB320002), Research grant from Nanjing Medical University and Southeast University (2017DN20) and Project from Nanjing Municipal Committee of Science and Technology (201803044).

\section{Author details}

'Jiangsu Key Laboratory of Oral Disease, Nanjing Medical University, Nanjing 210029, PR China. ${ }^{2}$ Department of Oral and Maxillofacial Surgery, Affiliated Stomatological Hospital, Nanjing Medical University, Nanjing 210029, PR China. ${ }^{3}$ Department of Oral Pathology, Affiliated Stomatological Hospital, Nanjing Medical University, Nanjing 210029, PR China

\section{Author contributions}

J.L. and Z.L. performed the experimental study, data collection and analysis and manuscript writing. Y.W., Y.W., D.W., and H.Y. carried out some animal experiments. W.Z., J.Y., and X.S. performed histological and statistical analyses. H.J. and J.Y. performed surgeries and follow-up. J.C. conceived and supervised the whole project. All authors have read and approved the final manuscript.

\section{Competing interests}

The authors declare that they have no competing interests.

\section{Publisher's note}

Springer Nature remains neutral with regard to jurisdictional claims in published maps and institutional affiliations.

Supplementary Information accompanies this paper at (https://doi.org/ 10.1038/s41419-019-1838-0).

Received: 25 March 2019 Revised: 23 July 2019 Accepted: 26 July 2019 Published online: 09 August 2019

\section{References}

1. Chi, A. C., Day, T. A. \& Neville, B. W. Oral cavity and oropharyngeal squamous cell carcinoma-an update. CA Cancer J. Clin. 65, 401-421 (2015).

2. Bray, F. et al. Global cancer statistics 2018: GLOBOCAN estimates of incidence and mortality worldwide for 36 cancers in 185 countries. CA Cancer J. Clin. $\mathbf{6 8}$, 394-424 (2018).

3. Chinn, S. B. \& Myers, J. N. Oral cavity carcinoma: current management, controversies, and future directions. J. Clin. Oncol. 33, 3269-3276 (2015).

4. Sacco, A. G. \& Cohen, E. E. Current treatment options for recurrent or metastatic head and neck squamous cell carcinoma. J. Clin. Oncol. 33, 3305-3313 (2015).
5. Ringash, J. Survivorship and quality of life in head and neck cancer. J. Clin. Oncol. 33, 3322-3327 (2015).

6. Carrillo, J. F. et al. Retrospective cohort study of prognostic factors in patients with oral cavity and oropharyngeal squamous cell carcinoma. Head. Neck $\mathbf{3 8}$, 536-541 (2016).

7. Reid, P. A. Wilson, P., Li, Y., Marcu, L. G. \& Bezak, E. Current understanding of cancer stem cells: Review of their radiobiology and role in head and neck cancers. Head. Neck 39, 1920-1932 (2017).

8. Beck, B. \& Blanpain, C. Unravelling cancer stem cell potential. Nat. Rev. Cancer 13, 727-738 (2013).

9. Birkeland, A. C., Owen, J. H. \& Prince, M. E. Targeting head and neck cancer stem cells: current advances and future challenges. J. Dent. Res 94, 1516-1523 (2015).

10. Prince, M. E. et al. Identification of a subpopulation of cells with cancer stem cell properties in head and neck squamous cell carcinoma. Proc. Natl Acad. Sci. USA 104, 973-978 (2007).

11. Boumahdi, S. et al. SOX2 controls tumour initiation and cancer stem-cell functions in squamous-cell carcinoma. Nature 511, 246-250 (2014).

12. Chen, D. et al. Targeting BMl1(+) cancer stem cells overcomes chemoresistance and inhibits metastases in squamous cell carcinoma. Cell Stem Cell $\mathbf{2 0}$ 621-634 e626 (2017).

13. Basu-Roy, U. et al. Sox2 maintains self renewal of tumor-initiating cells in osteosarcomas. Oncogene 31, 2270-2282 (2012).

14. Siegle, J. M. et al. SOX2 is a cancer-specific regulator of tumour initiating potential in cutaneous squamous cell carcinoma. Nat. Commun. 5, 4511 (2014).

15. Basu-Roy, U. et al. Sox2 antagonizes the Hippo pathway to maintain stemness in cancer cells. Nat. Commun. 6, 6411 (2015).

16. Keysar, S. B. et al. Regulation of Head and Neck Squamous Cancer Stem Cells by PI3K and SOX2. J Natl Cancer Inst 109, https://doi.org/10.1093/jnci/djw189 (2017).

17. Chou, M. Y., Hu, F. W., Yu, C. H. \& Yu, C. C. Sox2 expression involvement in the oncogenicity and radiochemoresistance of oral cancer stem cells. Oral. Oncol. 51, 31-39 (2015).

18. Lee, S. H. et al. SOX2 regulates self-renewal and tumorigenicity of stem-like cells of head and neck squamous cell carcinoma. Br. J. Cancer 111, 2122-2130 (2014).

19. Deng, P. et al. AFF4 promotes tumorigenesis and tumor-initiation capacity of head and neck squamous cell carcinoma cells by regulating SOX2. Carcinogenesis 39, 937-947 (2018).

20. Harvey, K. F., Zhang, X. \& Thomas, D. M. The Hippo pathway and human cancer. Nat. Rev. Cancer 13, 246-257 (2013).

21. Zanconato, F., Cordenonsi, M. \& Piccolo, S. YAP/TAZ at the roots of cancer. Cancer Cell 29, 783-803 (2016)

22. Cordenonsi, M. et al. The Hippo transducer TAZ confers cancer stem cellrelated traits on breast cancer cells. Cell 147, 759-772 (2011).

23. Li, Z. et al. The Hippo transducer TAZ promotes epithelial to mesenchymal transition and cancer stem cell maintenance in oral cancer. Mol. Oncol. $\mathbf{9}$, 1091-1105 (2015).

24. Chang, C. et al. A laminin 511 matrix is regulated by TAZ and functions as the ligand for the alpha6Bbeta1 integrin to sustain breast cancer stem cells. Genes Dev. 29, 1-6 (2015).

25. Bhat, K. P. et al. The transcriptional coactivator TAZ regulates mesenchymal differentiation in malignant glioma. Genes Dev. 25, 2594-2609 (2011).

26. Seo, E. et al. SOX2 regulates YAP1 to maintain stemness and determine cell fate in the osteo-adipo lineage. Cell Rep. 3, 2075-2087 (2013).

27. Zhang, W. et al. TEAD4 overexpression promotes epithelial-mesenchymal transition and associates with aggressiveness and adverse prognosis in head neck squamous cell carcinoma. Cancer Cell Int. 18, 178 (2018).

28. Bora-Singhal, N. et al. YAP1 regulates OCT4 activity and SOX2 expression to facilitate self-renewal and vascular mimicry of stem-like cells. Stem Cells $\mathbf{3 3}$ 1705-1718 (2015)

29. Maurizi, G., Verma, N., Gadi, A., Mansukhani, A. \& Basilico, C. Sox2 is required for tumor development and cancer cell proliferation in osteosarcoma. Oncogene 37, 4626-4632 (2018)

30. Wei, Z. et al. Overexpression of Hippo pathway effector TAZ in tongue squamous cell carcinoma: correlation with clinicopathological features and patients' prognosis. J. Oral. Pathol. Med 42, 747-754 (2013).

31. Chien, C. S. et al. Lin28B/Let-7 regulates expression of Oct4 and Sox2 and reprograms oral squamous cell carcinoma cells to a stem-like state. Cancer Res 75, 2553-2565 (2015) 
32. Chan, S. W. et al. TEADs mediate nuclear retention of TAZ to promote oncogenic transformation. J. Biol. Chem. 284, 14347-14358 (2009).

33. Liu-Chittenden, Y. et al. Genetic and pharmacological disruption of the TEADYAP complex suppresses the oncogenic activity of YAP. Genes Dev. 26, 1300-1305 (2012).

34. Rajbhandari, P. et al. Cross-cohort analysis identifies a TEAD4-MYCN positive feedback loop as the core regulatory element of high-risk neuroblastoma. Cancer Discov. 8, 582-599 (2018).

35. Verma, N. K. et al. Myeloid zinc finger 1 and GA binding protein co-operate with Sox2 in regulating the expression of yes-associated protein 1 in cancer cells. Stem Cells 35, 2340-2350 (2017).

36. Huang, C. F., Xu, X. R., Wu, T. F., Sun, Z. J. \& Zhang, W. F. Correlation of ALDH1, CD44, OCT4 and SOX2 in tongue squamous cell carcinoma and their association with disease progression and prognosis. J. Oral. Pathol. Med 43 492-498 (2014)

37. Zullig, L. et al. High sex determining region Y-box 2 expression is a negative predictor of occult lymph node metastasis in early squamous cell carcinomas of the oral cavity. Eur. J. Cancer 49, 1915-1922 (2013).

38. Fu, T. Y. et al. Association of OCT4, SOX2, and NANOG expression with oral squamous cell carcinoma progression. J. Oral. Pathol. Med. 45, 89-95 (2016).

39. Bayo, P. et al. Loss of SOX2 expression induces cell motility via vimentin upregulation and is an unfavorable risk factor for survival of head and neck squamous cell carcinoma. Mol. Oncol. 9, 1704-1719 (2015).

40. Schrock, A. et al. Expression and role of the embryonic protein SOX2 in head and neck squamous cell carcinoma. Carcinogenesis 35, 1636-1642 (2014).
41. Lei, Q. Y. et al. TAZ promotes cell proliferation and epithelial-mesenchymal transition and is inhibited by the hippo pathway. Mol. Cell Biol. 28, 2426-2436 (2008).

42. Li, Z. et al. Oncogenic roles of Bmi1 and its therapeutic inhibition by histone deacetylase inhibitor in tongue cancer. Lab Invest 94, 1431-1445 (2014)

43. Wang, Y. et al. The histone demethylase LSD1 is a novel oncogene and therapeutic target in oral cancer. Cancer Lett. 374, 12-21 (2016).

44. $\mathrm{Wu}, \mathrm{Y}$. et al. Therapeutic targeting of BRD4 in head neck squamous cell carcinoma. Theranostics 9, 1777-1793 (2019).

45. Wichmann, G. et al. The role of HPV RNA transcription, immune responserelated gene expression and disruptive TP53 mutations in diagnostic and prognostic profiling of head and neck cancer. Int J. Cancer 137, 2846-2857 (2015).

46. Zhou, $C$ et al. DNA methylation biomarkers for head and neck squamous cell carcinoma. Epigenetics 13, 398-409 (2018).

47. Renaud, G., Stenzel, U., Maricic, T., Wiebe, V. \& Kelso, J. deML: robust demultiplexing of Illumina sequences using a likelihood-based approach. Bioinformatics 31, 770-772 (2015).

48. Kendall, W. L., Pollock, K. H. \& Brownie, C. A likelihood-based approach to capture-recapture estimation of demographic parameters under the robust design. Biometrics 51, 293-308 (1995).

49. Lohavanichbutr, P. et al. A 13-gene signature prognostic of HPVnegative OSCC: discovery and external validation. Clin. Cancer Res 19 1197-1203 (2013) 